\title{
РАЗДЕЛ ХІV. ТЕЛЕКОММУНИКАЦИИ
}

\author{
Дахкильгова К.Б., Идигова А.А. \\ Экономическая модель конвергентного MVNO
}

ФГБОУ ВО Чеченский государственный университет

(Россия, Грозный)

doi: $10.18411 / 1 j-10-2020-54$

idsp: ljournal-10-2020-54

\section{Аннотация}

В статье дается краткий анализ текущего состояния рынка MVNO, экономическая модель конвергентного MVNO, приводится классификация. Также приводится анализ преимуществ развертывания работы таких операторов на территории России и рассматриваются перспективные инфокоммуникационные услуги для различных отраслей.

Ключевые слова: виртуальный оператор сотовой связи, инфокоммуникационные услуги, оператор связи, гипер-конвергентная инфраструктура.

\section{Abstract}

The article provides a brief analysis of the current state of the MVNO market, an economic model of a converged MVNO, and a classification. It also provides an analysis of the advantages of deploying such operators on the territory of Russia and considers promising infocommunication services for various industries.

Keywords: mobile virtual network operator, communication service, mobile network operator, HCI.

Виртуальный оператор сотовой связи (Mobile Virtual Network Operator, MVNO) это компания, предоставляющая своим абонентам услуги сотовой связи, однако не обладающая всеми признаками операторов реальных (например, собственной сетевой инфраструктурой, лицензией на предоставление услуг сотовой связи и т.д.). Деятельность виртуальных операторов возможна за счет соглашений c hostоператором, который предоставляет собственный трафик таким компаниям. При этом доход виртуального оператора складывается из разницы между платежами абонентов и платой реальному оператору сотовой связи (MNO - Mobile Network Operator) за предоставляемый трафик.

В самом примитивном случае у виртуальных операторов отсутствуют даже биллинговые системы подсчета абонентского трафика - это прерогатива вышестоящего оператора, предоставляющего трафик, либо отдается на аутсорсинг специализированным сервисным компаниям-посредникам между MVNO и MNO, так называемым MVNE - Mobile Network Operator Enables. Во-вторых, отличительной особенностью виртуальных операторов является гораздо большая гибкость по сравнению с глобальными игроками.

Отсутствие необходимости в приобретении лицензий на предоставление услуг сотовой связи побуждает использовать опыт виртуальных операторов при выходе на другие рынки. Развертывание собственной инфраструктуры при этом не требуется, что еще более повышает привлекательность MVNO.

Как пример, перспективная модель конвергентного MVNO для корпоративного оператора крупной промышленной корпорации в нефтегазовой отрасли.

Конвергенция фиксированных и мобильных сетей FMC (Fixed Mobile Convergence) - это объединение различных типов сетей на единой технологической 
основе (рис. 1). Это существенно расширяет возможности услуг MVNO, которые ранее строились на раздельной основе.

В последние годы тенденцией операторского бизнеса стала т.н. «цифровая трансформация», которая еще более обогатила возможности конвергентных сетей. Суть цифровой трансформации - переход от «аппаратного» способа построения сетей (когда каждая сетевая функция реализуется при помощи выделенного аппаратного устройства) на программную реализацию, когда функции сети реализуются при помощи программных модулей, работающих на стандартном вычислительном оборудовании в центрах обработки данных ЦОД (дата-центры).

При этом слово «виртуальный» меняет смысл: если раньше виртуальным называли оператора, который не имеет собственной радиосети, то теперь имеется в виду «виртуализация сетевых функций» NFV (Network Function Virtualization). Появился даже термин «виртуальный MVNO» (vMVNO), в котором компоненты сетевой операторской инфраструктуры реализованы на стандартном ИТ-оборудовании дата-центров (серверы, системы хранения, и локальные сети, их соединяющие).
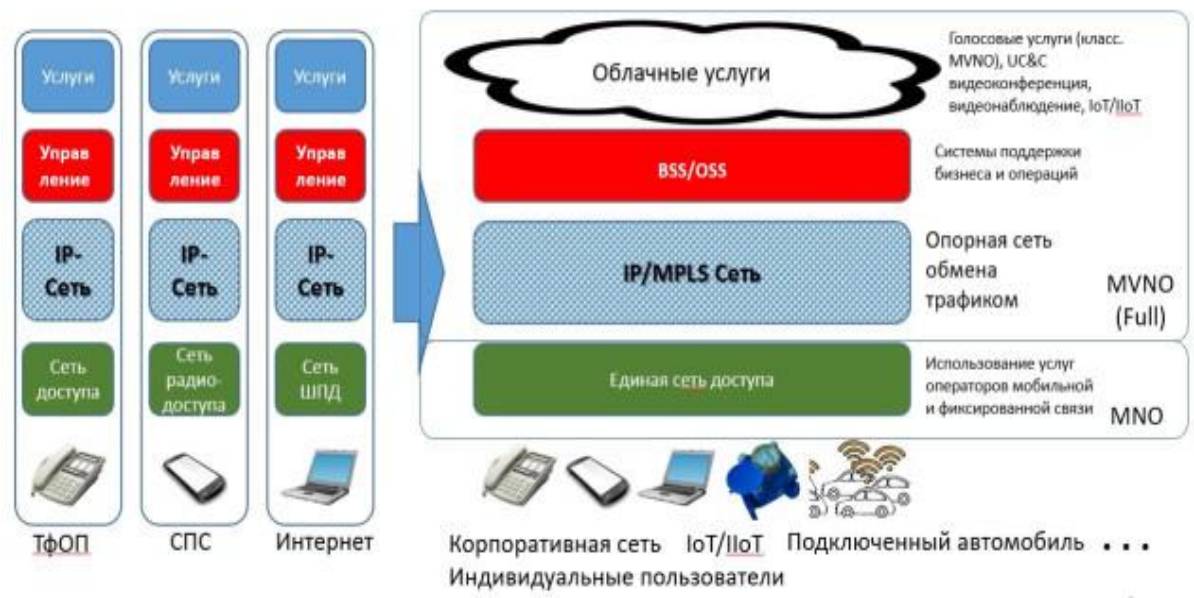

Рис. 1. Конвергенция фиксированных и мобильных сетей и расширение возможностей услуг

Смысл перехода с традиционных аппаратных платформ на виртуальные ИТплатформы в том, что при этом удается достичь снижения затрат на построение и техническую эксплуатацию сети, стандартизации и унификации сетевого оборудования, расширения спектра услуг сети, снижения времени разработки и вывода новых услуг на рынок.

Общая архитектура конвергентного MVNO показана на рис. 2.

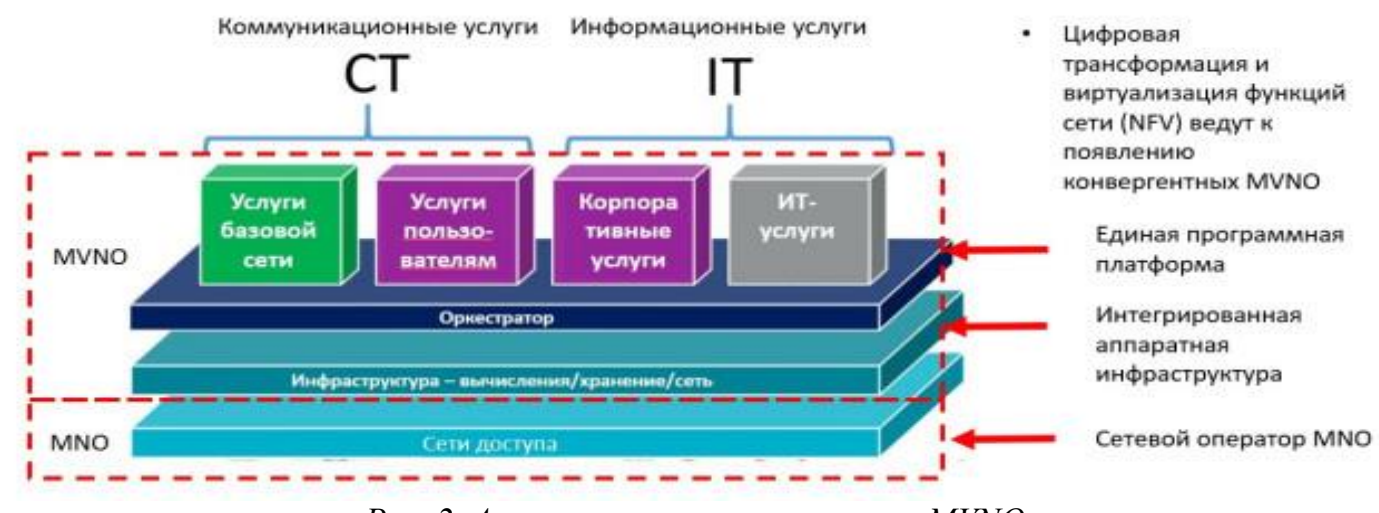

Рис. 2. Архитектура конвергентного MVNO

Три стандартных компонента инфраструктуры (как для обычных операторов, так и виртуальных), это: вычисления (server), хранение данных (storage) и сеть 
(network). Эти компоненты могут быть реализованы как в виде раздельного оборудования в дата-центре, так и виде т.н. «Гипер-конвергентной инфраструктуры» HCI (Hyper-Converged Infrastructure). То есть, мало того, что конвергентной становится архитектура сети на основе технологии NFV, так еще и слой инфраструктуры на рис. 3 также подвержен процессу конвергенции, и, в этом случае, к слову «конвергенция» добавляется еще и «гипер».
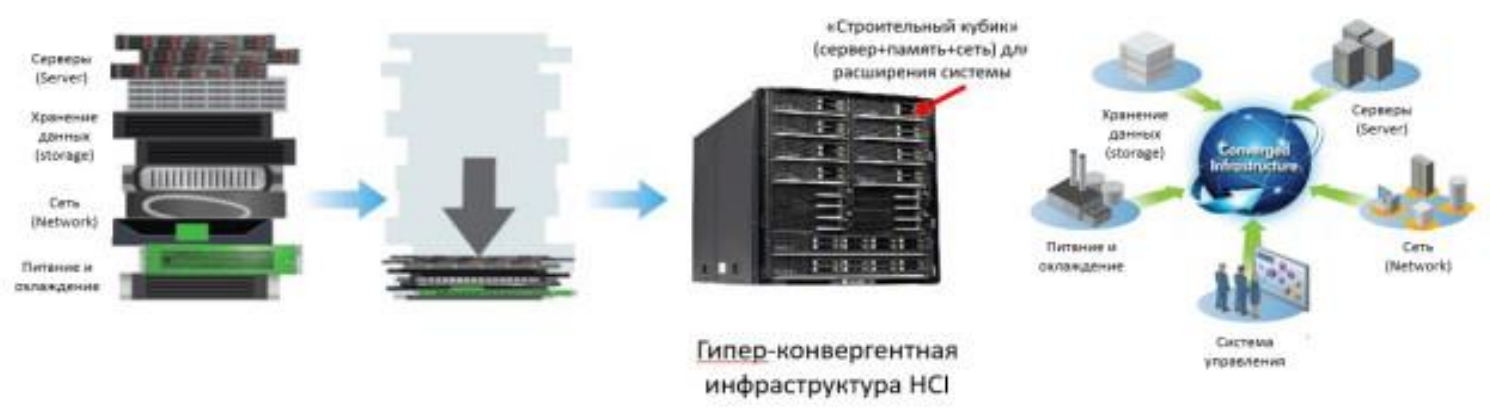

Рис. 3. Гипер-конвергентная инфраструктура

Гипер-конвергентная инфраструктура НCI занимает все большую долю в создании дата-центров и корпоративных сетей, а также в создании инфраструктуры операторов MNO/MVNO.

Их применение позволяет значительно упростить строительство инфраструктуры, которая создается из «стандартных строительных блоков» и может плавно наращиваться в соответствии с потребностями. Начальные инвестиции заказчика могут быть значительно сэкономлены, поскольку отпадает необходимость проектировать и строить инфраструктуру оператора «с запасом» под будущее развитие.

— Конвергентные услуги унифицированной связи и коллективной работы UC\&C (Unified Communication \& Collaboration)

Если широко известная услуга FMC представляет собой гибрид между фиксированной и мобильной связью (например, в зоне Wi-Fi сотовый телефон может выполнять роль фиксированного), то услуга UC\&C может предоставить гораздо больший функционал.

Фактически, UC\&C предоставляет все услуги корпоративной сети для офисной работы на единой платформе.

— «Видеонаблюдение как услуга» VSaaS (Video Surveillance as a Service).

При традиционном способе видео должно накапливаться и/или анализироваться на серверах, установленных на предприятии, с возможностью просмотра требуемых фрагментов через Интернет на терминалах уполномоченного персонала. При облачном видеонаблюдении VSaaS на предприятии устанавливаются только видеокамеры, которые подключаются к платформе конвергентного MVNO через каналы мобильной сети. На рынке имеется достаточный выбор таких видеокамер. Таким образом, расходы на развёртывание системы видеонаблюдения значительно снижаются.

- Система поддержки бизнеса как услуга BSSaaS (Business Support System-as-a-Service)

Система поддержка бизнеса BSS (Business Support System) - важная часть организационной структуры операторов, как MNO, так и MVNO. В классическом MVNO оператора система BSS арендуется у одного из базовых операторов. Вывод собственной BSS в облако конвергентного MVNO позволяет достичь существенной экономии операционных расходов бизнеса корпорации. Облачные системы BSS можно использовать и в других отраслях: ЖКХ, управления городским транспортом, такси, сетями быстрого питания, гостиничными сетями и т.д. 
— Услуги информационной безопасности конвергентного MVNO

Информационная безопасность в конвергентном MVNO может быть обеспечена централизованно на единой платформе «безопасность как услуга» SECaaS (Security-asa-Service). При этом значительно повышается уровень защиты для корпоративных пользователей, дочерних компаний и филиалов, а также внешних пользователей услуг связи и Интернета.

\section{— Услуги профессиональной мобильной радиосвязи (ПMP) eLTE}

Технология еLTE обеспечивает возможность беспроводной широкополосной передачи данных с пиковой скоростью 50 Мбит/с из сети и 20 Мбит/с в сеть при полосе 5, 10 и 15 МГц. Система рассчитана для применения в вертикальных отраслях, таких как госорганы, транспорт, электроэнергетика, добыча и переработка полезных ископаемых, крупные предприятия и т. д. Кроме того, возможно применения eLTE в индустриальном Интернете вещей (IIоT).

\section{— Услуги Интернета вещей IoT (Internet of Things)}

Перспективным является применение ІоТ в проектах «умного здания» и в системах интеллектуального управления электроснабжением.

\section{— Экономика конвергентного MVNO}

Аналитическая компания IDC в августе 2015 года провела опрос 13 организаций с численностью сотрудников от 200 до 18 тыс. чел., внедривших гипер-конвергентную инфраструктуру HCI компании Nutanix. По данным исследования, для таких относительно небольших предприятий средняя экономия в год за счет уплотнения инфраструктуры конвергентного оборудования составила почти 2,22 млн. долларов.

Следует отметить, что в статье рассматривалась только экономия за счет применения гипер-конвергентной инфраструктуры HCI, что можно достаточно просто оценить. Однако, если учитывать доходы от услуг конвергентного MVNO, спектр которых значительно шире, чем услуги «классического» MVNO оператора, то экономическая эффективность модели конвергентного MVNO окажется гораздо выше.

Таким образом, собственный MVNO доступен практически любому бизнесу, готовому получить перспективный источник дохода и надёжный инструмент работы с клиентами.

MVNO - не конкуренты традиционным операторам, а партнеры. Выстраивание взаимодействия по стратегии «win-win» со своим базовым оператором играет ключевую роль для MVNO-оператора. Дело в том, что в структуре затрат любого традиционного оператора немалую роль играют привлечение и обслуживание клиентов, реклама и организация приема платежей. Кроме того, поскольку рынок насыщен, борьба между традиционными операторами идет не за новых клиентов, а за увеличение своей доли на рынке - очевидно, за счет переманивания абонентов у конкурентов.

В целом перспективы MVNO в России неоднозначны и будут во многом определяться действиями отраслевого регулятора. Основным движущим фактором рынка может стать осознание мобильными операторами преимуществ использования модели MVNO и нахождение для себя оптимальных наборов бизнес-стратегий при работе с крупными нишевыми сегментами потребителей.

$$
\text { *** }
$$

1. Какие виртуальные операторы связи MVNO есть в России. [Электронный ресурс]. - Режим доступа: URL: https://telecomtimes.ru/2018/11/mvno-in-russia/

2. ИТ-портал компании «Инфосистемы Джет». [Электронный ресурс]. - Режим доступа: URL: http://www.jetinfo.ru/stati/mvno-sinergiya-osnovnogo-biznesa-i-telekomuslug

3. Виртуальные операторы и российская реальность. [Электронный ресурс]. - Режим доступа: URL: https://www.computerra.ru/230006/virtualnye-operatory-i-rossijskaya-realnost/ 
4. Информационно-аналитический портал, посвящённый отечественным телекоммуникациям в целом, интернет-провайдингу, строительству сетей, передаче и хранению данных. [Электронный peсурс]. - Режим доступа: URL: https://nag.ru/go/text/101183/

5. Независимое российское онлайн-издание, посвященное цифровым технологиям. [Электронный peсурс]. - Режим доступа: URL: https://3dnews.ru/570434

6. Крупнейшая в Рунете площадка для предпринимателей. [Электронный ресурс]. - Режим доступа: URL: https://vc.ru/finance/67047-chto-nam-stoit-mvno-postroit

7. MCN Telecom - первый мобильный виртуальный оператор (MVNO) для бизнеса. [Электронный peсурс]. - Режим доступа: URL: https:/telcojournal.mcn.ru/interview/full-light-mvno-tekhnologiyamulti-sim-perspektivy

\section{Дахкильгова К.Б., Идигова А.А. \\ Проблемы пространственного поиска и обнаружения в системах квантовой криптографии \\ ФГБОУ ВО Чеченский государственный университет \\ (Россия, Грозньй)}

doi: $10.18411 / 1 j-10-2020-55$

idsp: ljournal-10-2020-55

Аннотация

В статье рассматриваются проблемы поиска, приёма, обнаружения и обработки оптических сигналов, которые важны в системах квантовой криптографии, интенсивно развивающихся в настоящее время.

Ключевые слова: фотоны, однофотонный импульс, пространственный поиск, одноканальная регистрация, защита информации, квантовая криптография.

\section{Abstract}

The article discusses the problems of searching, receiving, detecting and processing optical signals, which are important in quantum cryptography systems, which are currently intensively developing.

Keywords: photon, single photon pulse, existential search, the single-channel registration, cybersecurity, quantum cryptography.

Проблема поиска, приёма, обнаружения и обработки оптических сигналов важна в системах квантовой криптографии, которые интенсивно развиваются в настоящее время.

Привлекательность идеи квантовой криптографии состоит в том, что предложен новый метод создания случайного секретного ключа между пользователями квантовой линии связи. Его абсолютная секретность и невозможность незаметного прослушивания посторонним лицом основана на законах квантовой физики в противоположность используемым сейчас методам криптографии, которые основаны на математических закономерностях и, в принципе, поддаются расшифровке.

При переходе от сигналов, где информация кодируется импульсами, содержащими тысячи фотонов, к сигналам, где среднее число фотонов, приходящихся на один импульс, много меньше единицы (порядка 0,1 ), вступают в действие законы квантовой физики. Именно на использовании этих законов в сочетании с процедурами классической криптографии основана природа секретности, обеспечиваемая квантовой криптографией. Здесь применим принцип неопределённости Гейзенберга, согласно которому попытка произвести измерения в квантовой системе вносит в неё нарушения, и полученная в результате такого измерения информация квалифицируется принимаемой стороной как дезинформация.

Интенсивные работы проводятся по созданию атмосферных и спутниковых систем КРК. Действительно, создание глобальной телекоммуникационной сети 